\title{
The NASA Wallops Arc-Second Pointer (WASP) System for Precision Pointing of Scientific Balloon Instruments and Telescopes
}

\author{
David W. Stuchlik ${ }^{1}$ and Raymond J. Lanzi ${ }^{2}$ \\ NASA GSFC Wallops Flight Facility, Wallops Island, VA, 23337
}

\begin{abstract}
The National Aeronautics and Space Administration's (NASA) Wallops Flight Facility (WFF), part of the Goddard Space Flight Center (GSFC), has developed a unique pointing control system for instruments aboard scientific balloon gondolas. The ability to point large telescopes and instruments with arc-second accuracy and stability is highly desired by multiple scientific disciplines, such as Planetary, Earth Science, Heliospheric and Astrophysics, and the availability of a standardized system supplied by NASA alleviates the need for the science user to develop and provide their own system. In addition to the pointing control system, a star tracker has been developed with both daytime and nighttime capability to augment the WASP and provide an absolute pointing reference. The WASP Project has successfully completed five test flights and one operational science mission, and is currently supporting an additional test flight in 2017 , along with three science missions with flights scheduled between 2018 and 2020. The WASP system has demonstrated precision pointing and high reliability, and is available to support scientific balloon missions.
\end{abstract}

\section{Introduction}

$\mathrm{T}$ HE WASP consists of a pointing control system (PCS) and a star tracker subsystem. The pointing system includes several standardized mechanical assemblies and electronics subsystems. It is designed to work in conjunction with the standard NASA gondola rotator, which is an azimuth control device that is located above the gondola which provides rough azimuth positioning with an accuracy of approximately one degree. The other components of the PCS are an inner and outer gimbal frame, a set of four WASP hubs, a fiber optic gyroscope, the avionics susbsystem, a trim balance subsystem, and a latch mechanism. All of these subsystems have successfully demonstrated their capabilities on multiple flights, and have a standardized design that can be replicated and used without any new engineering design effort. The star tracker, known as the Camera Attitude Reference Determination System (CARDS), consists of a commercial camera head and lens, commercial processor with custom software, and a baffle for stray light rejection. In 2016, the WASP PCS and CARDS were used for the first time operationally on a science mission called X-Calibur, a hard X-ray telescope with a Principle Investigator (PI) from Washington University at St. Louis. X-Calibur is currently being prepared for an Antarctic Long Duration Balloon (LDB) mission, and the WASP Team is supporting two other science missions at the same time. The Planetary Imaging Concept Testbed Using a Recoverable Experiment - Coronagraph (PICTURE-C) first flight is scheduled for 2018 at either Fort Sumner, NM or Palestine, TX, and the Gondola for High Altitude Planetary Science (GHAPS) first flight is scheduled for 2020. The three science missions have completely different science objectives and demonstrate the versatility of the WASP system and the ability of the small WASP team at WFF to provide support efficiently.

\section{System Description}

The WASP PCS and CARDS subsystems were described in detail in a previous AIAA paper. ${ }^{1}$ The PCS works in conjuction with an electronically driven azimuth positioning system above the gondola, which is a standard NASA support system typically used to orient gondola solar panels toward the sun in flight. For WASP, the gondola rotator provides rough positioning toward the desired targets with an accuracy of approximately one degree. The other

\footnotetext{
${ }^{1}$ Aerospace Engineer, Balloon Program Office, Code 820.

${ }^{2}$ Aerospace Engineer, Guidance, Navigation and Control and Mission Systems Engineering Branch, Code 598.
}

American Institute of Aeronautics and Astronautics 
components of the WASP System are mounted on the gondola, and are located on either the external gondola frame or on the pointed structure.

The PCS uses two large gimbal frames to provide pitch and yaw articulation. The standard WASP frames are constructed of aluminmum box tubes riveted together. The outer gimbal frame supports the outer surfaces of the pitch control hubs, and the inner frame is attached to the inner surfaces of the pitch hubs and holds the yaw hubs which interface to the pointed structure (Fig.1). The gimbal frame hub mounts are machined with a tolerance of $+/-$ 0.001 inch to precisely align the hubs which minimizes the torque needed to control the system. The pointed structure is mounted within the inner frame, and is balanced in all three axes so the center of gravity is located exactly where the two hub axes intersect.

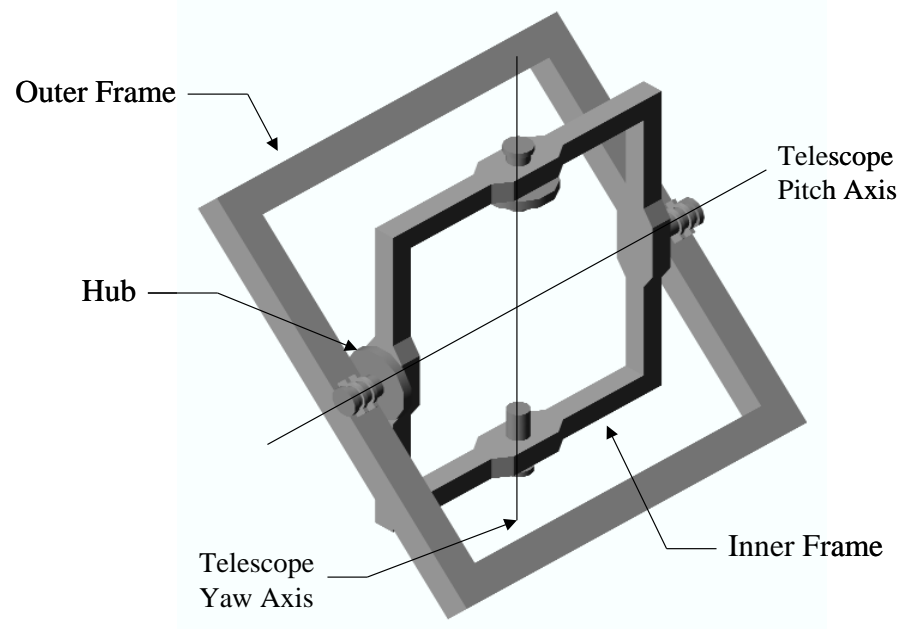

Figure 1: WASP Gimbal Frames

There are two types of hubs used by the PCS, the motor hubs and the resolver hubs. One of each is used for each axis. The hubs include a constantly rotating center shaft design which eliminates static friction in the bearings. The inner and outer halves of the hub are attached to the central shaft through four sets of bearings, and the shaft is continuously rotated by a small electric motor connected to a gearhead to reduce the rotation rate.

The WASP motor hubs include a large diameter brushless Direct Current (DC) torque motor, which provides the drive forces to the system. Figure 2 shows a resolver hub, which includes a standard brushless angle resolver which provides an angular measurement between the rotor and stator halves of the hub.

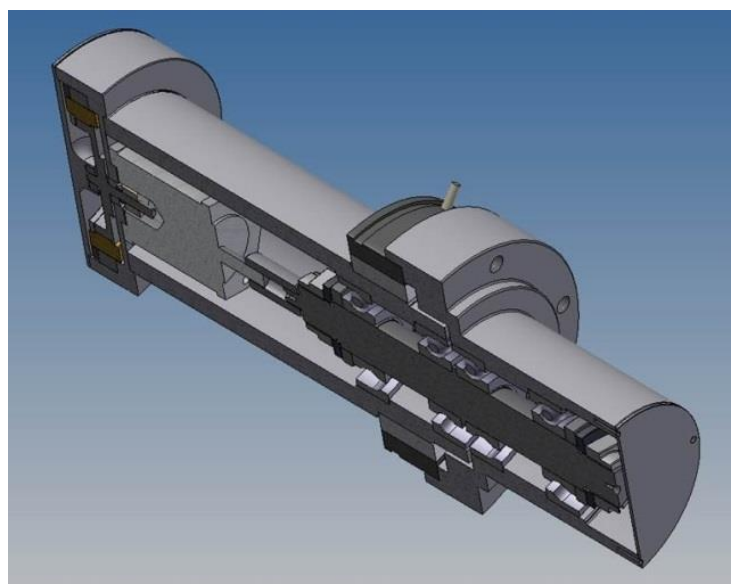

Figure 2: WASP Resolver Hub

The PCS also consists of a number of electronics subsystems that are typically mounted on the pointed structure. Some of the electronics are commercially procured including the PCS flight computer and the CARDS flight computer. The remainder of the PCS electronics are custom designed and fabricated to perform the various required functions. 
The fiber optic gyroscope is also a commercially procured item which provides position and acceleration outputs to the WASP flight computer for use by the control system algorithm. Instrument attitude is computed by integrating incremental angles (delta-Theta) output from the LN251 system. Control torques are computed using a modified Proportional-Integral-Derivative control law in each axis.

The remaining PCS subsystems consist of static and dynamic balance assemblies and the latch mechanism. The static balance assemblies are used to trim the balance prior to flight, and the dynamic balance subsystem consists of motor driven weights that can compensate for changes in pointed structure balance during the flight (Fig. 3). The latch mechanism secures the telescope, or other pointed structure, to the external gondola framework during the launch and ascent portions of the flight, releases the pointed structure when at float, and then engages and locks the structure in place prior to the termination of the flight. The system has full redundancy, with two independent methods for both uncaging and caging. (Fig. 4).

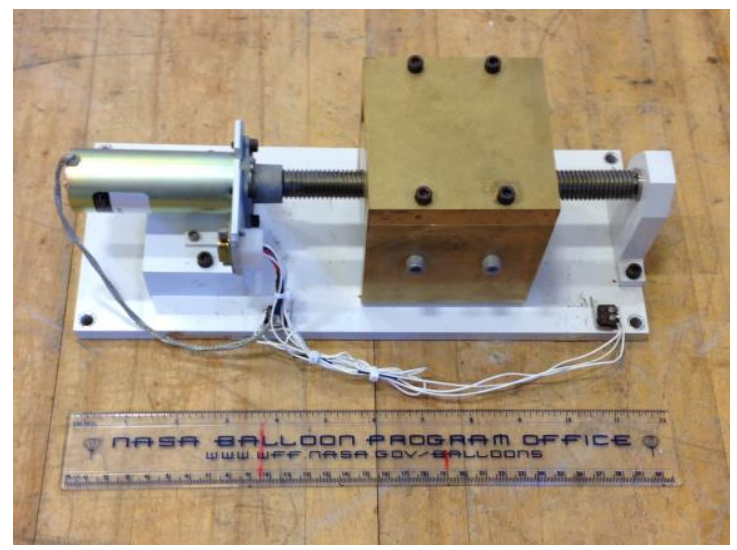

Figure 3: Dynamic Balance Weight

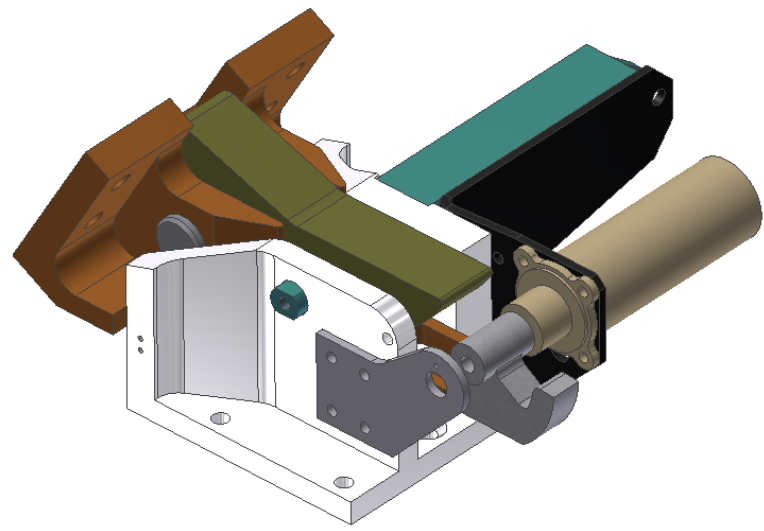

Figure 4: Caging System

The WASP CARDS star tracker camera subsystem includes a 2.8 megapixel camera, a C-mount lens with 100 $\mathrm{mm}$ focal length, and a custom baffle with an exclusion angle of approximately 20 degrees. The CARDS camera has a field of view of 5.9 degrees by 4.4 degrees, and has demonstrated ability to image stars dimmer than magnitude 5 during the daytime at balloon float altitude (Fig. 5). 


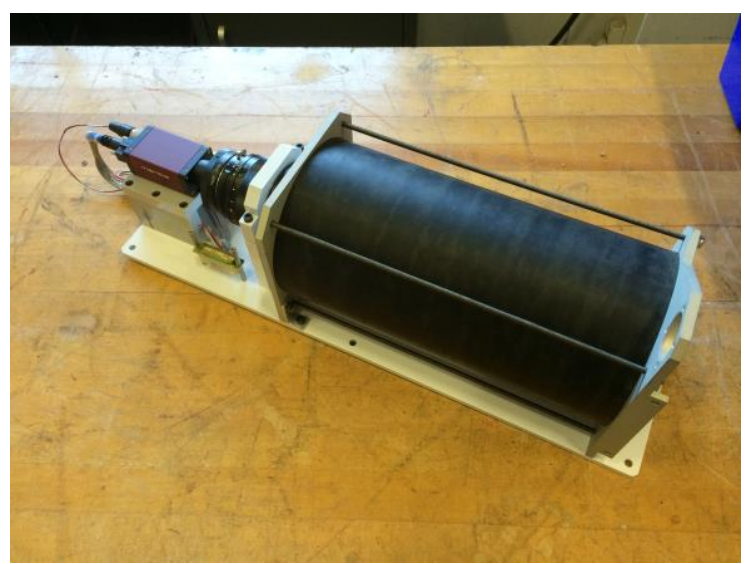

Figure 5: CARDS Star Tracker

\section{X-Calibur Mission}

A total of five WASP test flights were conducted from 2011 through 2014. All flights were conducted from the Fort Sumner, New Mexico launch site, and had flight durations ranging from 4 hours and 45 minutes to over 15 hours. Details of the gondola configurations and performance of the system are described in a previous AIAA paper $^{1}$, and tracking performance with pitch and yaw errors of less than one arc-second were observed.

In 2014, the WASP Team began a collaboration with the X-Calibur Science Team to provide the PCS and CARDS for their next two science missions. X-Calibur measures the linear polarization of hard X-rays in the energy range of $25-70 \mathrm{keV}$. To make these measurements, X-Calibur uses a 255 shell X-ray focusing mirror provided by Dr. Takashi Okajima at the NASA GSFC X-ray Astrophysics Laboratory, and a rotating polarimeter provided by Washington University in St. Louis (WUSTL). Dr. Henric Krawczynski at WUSTL is the Principal Investigator (PI) for this mission, and two flights were scheduled to conduct the science mission.

The pointed structure design for X-Calibur was challenging, as a very rigid structure was needed to maintain alignment between the focusing X-ray mirror and the detector located 8 meters away. The alignment between these two elements needed to be maintained during flight with an accuracy of better than 1 millimeter. The Science Team designed and developed a truss structure made of carbon fiber tubes and aluminum joints with honeycomb bulkheads at each end to support the science instruments and WASP subsystems (Fig. 6).

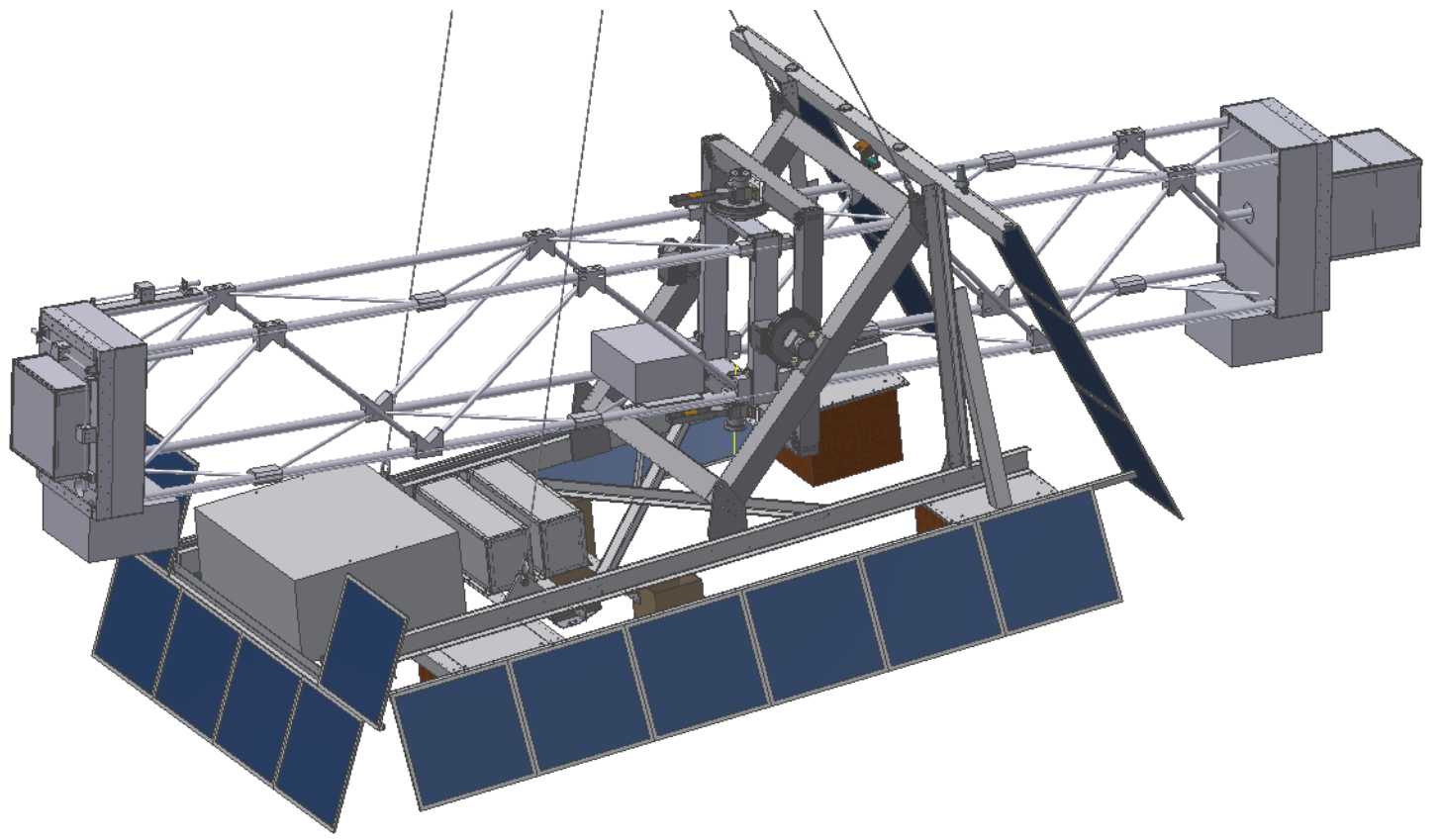

Figure 6: X-Calibur Truss and Gondola Design

4

American Institute of Aeronautics and Astronautics 
X-Calibur was designed to support two flight locations. The first flight was conducted from Fort Sumner, New Mexico, and used primary batteries as a power source, and used line of sight communications with ground stations for telemetry and commands. The second flight, scheduled for December 2018 in Antarctica, will use solar panels as show in Figure 6 to generate power, and will use satellite communications for telemetry and commands.

X-Calibur was launched on September 17, 2016 and had a total flight duration of 24 hours and 37 minutes. During the long flight, WASP was used to point at X-ray targets including Crab Nebula, Scorpius X-1, Cygnus X-1, and GRS1915+105. WASP provided excellent tracking capability with sub arc-second tracking errors similar to previous flights (Fig. 7). The WASP absolute pointing performance was not optimal due to misalignment between the star tracker and the inertial rate unit (IRU), and was further degraded during daytime by a high ambient light background which prevented the star tracker from consistently maintaining lock.
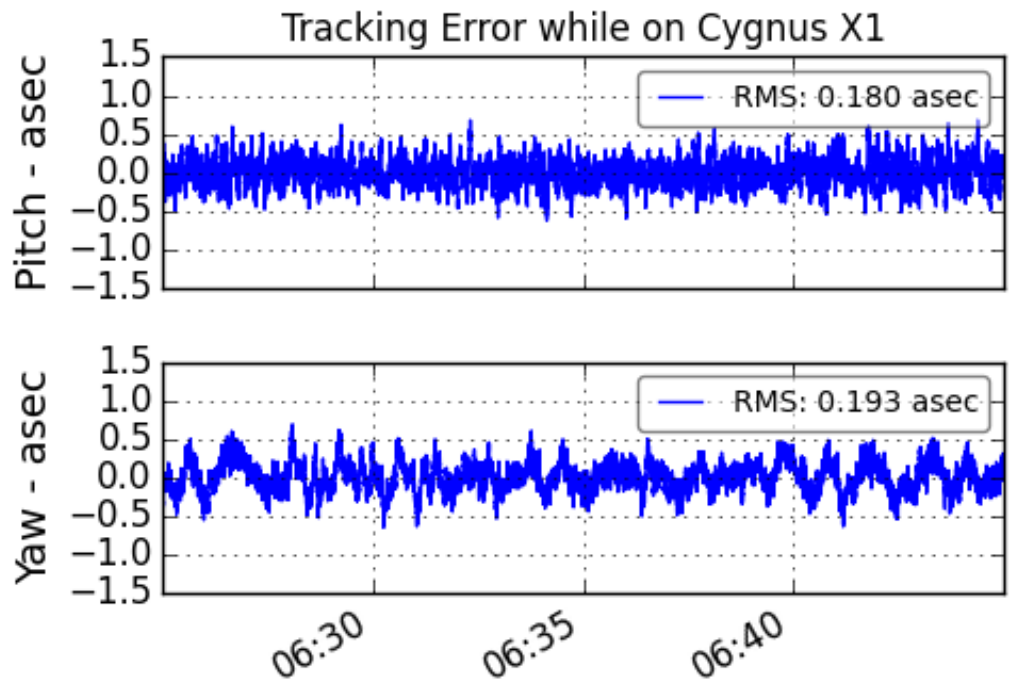

Figure 7: X-Calibur Tracking Error

The effects of the misalignment between the IRU and star tracker can be seen in Figure 8, which shows the residual differences between the star tracker and the Extended Kalman Filter (EKF) attitude solution. The oscillations seen in the CARDS residual errors coincide with roll motions around the boresight originating from the natural pendulous motions of the gondola, flight train and rotator motions. Roll coupling into the pitch and yaw axes indicates a misalignment between the star tracker and the IRU.
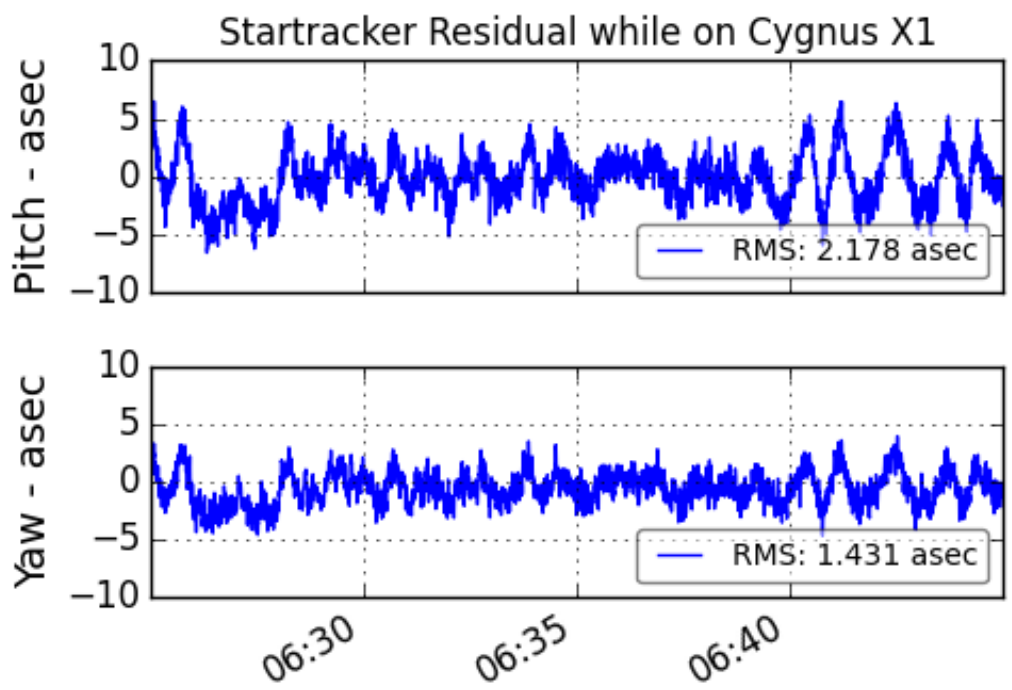

Figure 8: Star Tracker Residual Errors during X-Calibur Flight 5

American Institute of Aeronautics and Astronautics 
The misalignment was identified by post flight batch processing of five to ten minute segments of CARDS and IRU pointing data. Since the flight, a misalignment correction technique has been developed to eliminate the roll coupling, and ground testing has been successfully performed demonstrating the technique.

\section{Other Missions with WASP support}

In addition to the on-going support of X-Calibur, and the preparations for re-integration and the Antarctic long duration flight in December 2018, The WASP Team is supporting two other science missions, and a 2017 Test Flight effort.

\section{A. Planetary Imaging Concept Testbed Using a Recoverable Experiment - Coronagraph (PICTURE-C)}

The PICTURE-C mission plans to directly image and spectrally characterize dust and debris orbiting nearby stars with the possibility of discovering bright, gas giant exoplanets. The PI is Dr. Supriya Chakrabarti from University of Massachusettes at Lowell (UMASS Lowell). To characterize exoplanetary dust, PICTURE-C will use a 24 inch telescope and an instrument called a high-contrast coronagraph to directly image the inner 1 to 5 AU of nearby stellar systems. This instrument uses a focal plane mask and extreme adaptive optics to attenuate the starlight so that the faint glow of dust and planets can be seen.

The Preliminary Design Review (PDR) for PICTURE-C was conducted at Wallops on August 4, 2016, and the Critical Design Review (CDR) is scheduled for 2017. The gondola mechanical design (Fig. 9) uses the same external gondola structure design that was developed at WFF for X-Calibur, and the PCS makes use of all the standard mechanical and electronic subsystems flown on earlier flights. This demonstrates the cost effective and efficient approach that WASP can provide for science support.

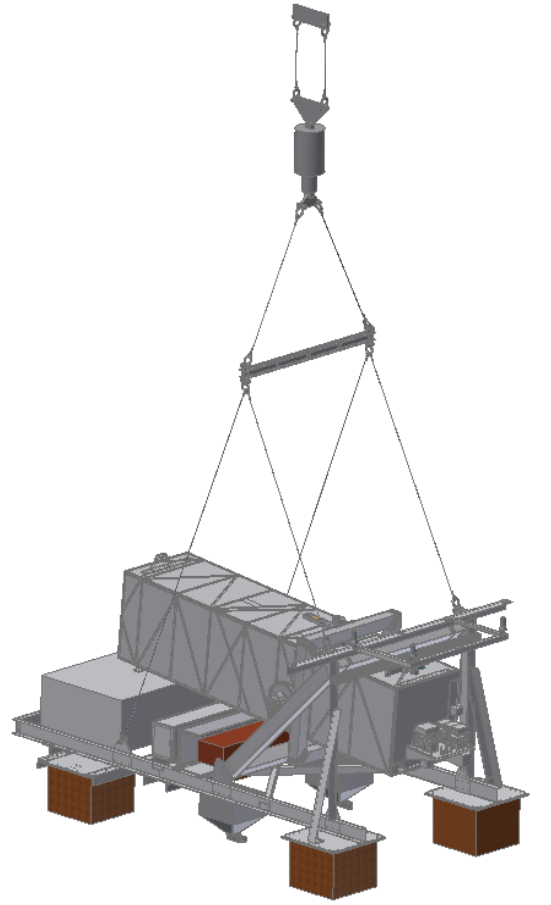

Figure 9: PICTURE-C Gondola Design

PICTURE-C plans to fly two balloon missions. The first flight is scheduled for 2018 from either Palestine, Texas or Fort Sumner, New Mexico. The second flight is planned for the fall of 2019 from Fort Sumner.

\section{B. Gondola for High Altitude Planetary Science (GHAPS)}

The other project currently being supported by WASP is GHAPS. This project is managed by Monica Hoffman at NASA Glenn Research Center (GRC) and includes an Optical Telescope Assembly (OTA) to be provided by NASA Marshall Space Flight Center (MSFC). GHAPS will provide a re-useable balloon platform to meet NASA Planetary Science goals and objectives as outlined in the NASA Decadal Survey. It includes a 1 meter diameter 
primary mirror which can be optimized for either infrared or ultraviolet and visible observations. GHAPS is designed for a minimum of five flights with low cost refurbishment between each flight (Fig. 10).

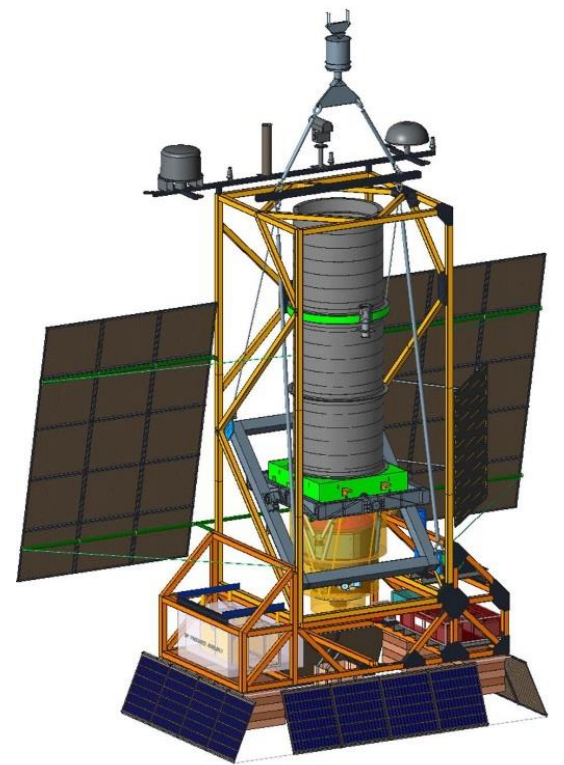

Figure 10: GHAPS Gondola Preliminary Design

One of the challenges of the GHAPS project is to design and develop a gondola with a large optical aperture that meets the stringent mass requirements to fly on a NASA Super Pressure Balloon (SPB). The total mass available for science flight hardware on a SPB is approximately $400 \mathrm{~kg}$. less than on a conventional zero-pressure balloon.

The WASP Team is making several design modifications to their standard systems to support the GHAPS configuration. The large primary mirror exceeds the diameter capability of the standard WASP inner and outer gondola frames, so an extra large (XL) frame has been designed to accommodate the OTA. In order to minimize the increase in mass of the larger frames, the inner gimbal frame will be constructed from machined aluminum instead of aluminum box tubes (Fig. 11). The larger frames will provide 1.34 meter of vertical clear space between the two WASP yaw hubs, where the WASP standard extended inner frame provides one meter vertical space.

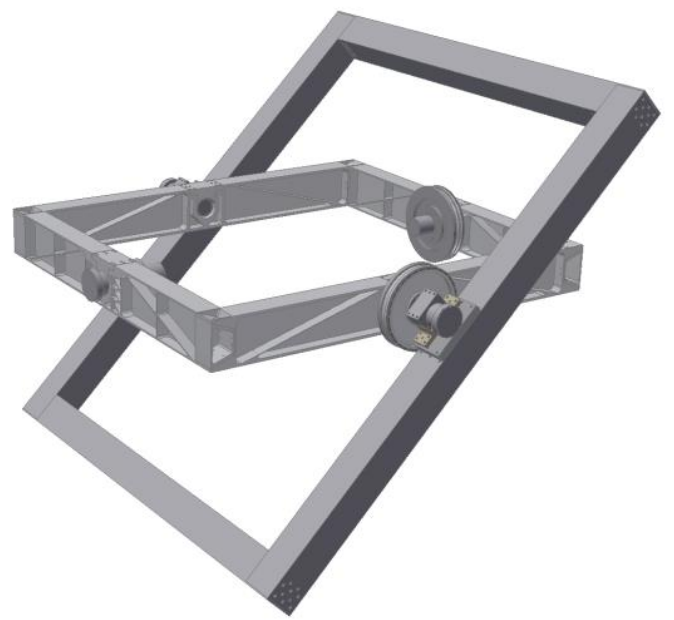

Figure 11: WASP Gimbal Frames for GHAPS

The WASP Avionics locations are also being modified to support GHAPS. Typically the PCS and CARDS flight computers are mounted on a main avionics deck located on the pointed structure along with the other control and monitoring electronics. For GHAPS, the project requested WASP to minimize the amount of electronics mounted on the OTA, which minimizes the thermal input to the telescope structure. As a result, the GHAPS configuration will consist of the CARDS flight computer, a video converter circuit, an H-Bridge driver circuit, and a housekeeping data acquisition circuit on the GHAPS OTA, which will reduce the power consumption of WASP components on 
the OTA by approximately 40 watts. The remainder of the WASP Avionics Deck mounted electronics will be mounted on the gondola external structure.

The other major change being made by WASP to support GHAPS is the star tracker baffle design. On previous flights the star tracker was mounted near the front edge of the pointed structure, and a small baffle is used to keep out stray light. The standard light baffle tube dimensions are approximately $15 \mathrm{~cm}$ in diameter and $30 \mathrm{~cm}$ in length. Due to the star tracker mounting location on the OTA, a much larger baffle tube approximately $40 \mathrm{~cm}$ in diameter and 1.2 meter in length is being designed.

\section{WASP 2017 Test Flight}

The 2017 Test Flight is scheduled for September in Fort Sumner, New Mexico, and will demonstrate improvements to the star tracker centrioding algorithm, and the misalignment correction technique. In addition, the flight will demonstrate the relocated electronics in the GHAPS configuration, and will also include the larger star tracker baffle design that is needed by GHAPS.

In order to minimize cost and development time, the same external gondola design used for X-Calibur and PICTURE-C will be used for the test flight. Also, the pointed structure will be based on the same $25.4 \mathrm{~cm}$ square steel tube that was used on the first two WASP test flights (Fig. 12).

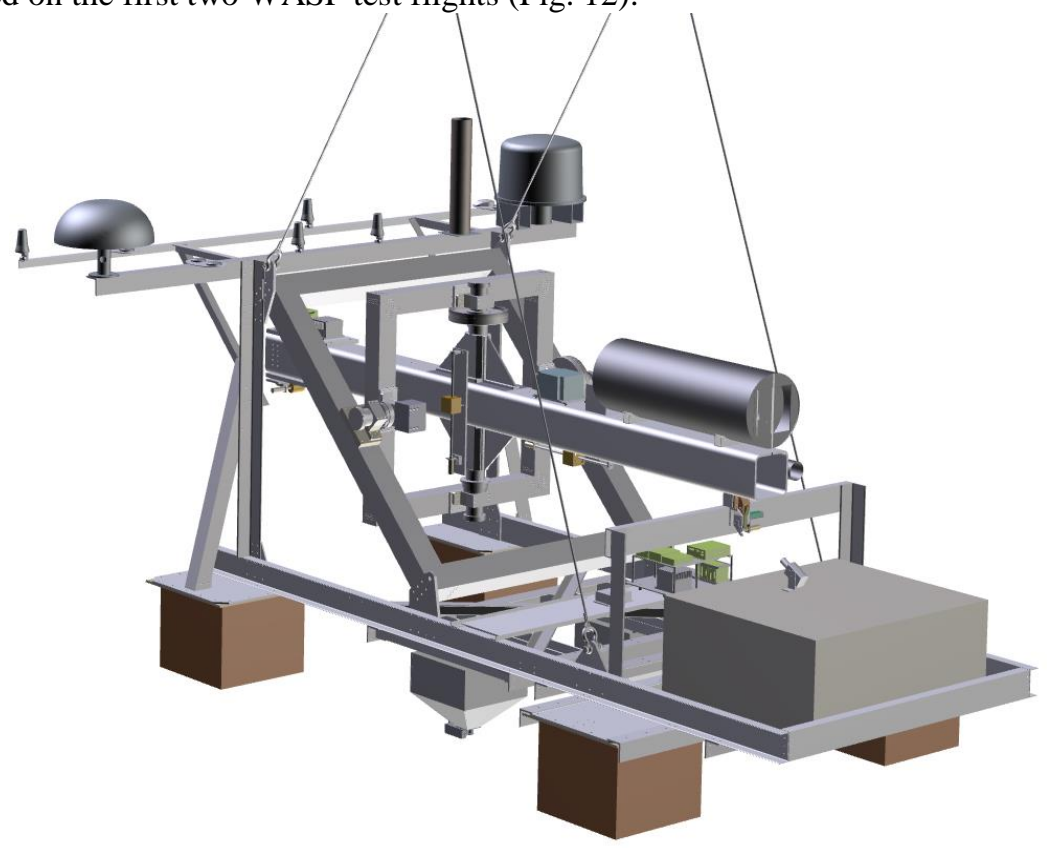

Figure 12: WASP 2017 Test Flight Design

\section{Acknowledgments}

The successful WASP Project is the result of the combined efforts of many people both within and outside NASA. The author would like to thank the WASP Project Team, which includes Wallops civil servants and contractors both on-site at Wallops and off. In addition, acknowledgment and gratitude would like to be expressed to the administrative, management, procurement, and logistics support personnel at WFF and elsewhere that assist with the project. The WASP Project Team would also like to thank the Science Teams that have collaborated with us, and appreciate the assistance and outstanding support from the CSBF personnel who helped make each launch and flight a success.

\section{References}

${ }^{1}$ Stuchlik, D. W., "The Wallops Arc Second Pointer - A Balloon Borne Fine Pointing System", AIAA Balloon Systems Conference, AIAA AVIATION Forum, (AIAA 2015-3039) 